\title{
Medición Del espacio fisCAL EN MÉXico
}

\author{
Héctor Juan Villarreal y Sunny A. Villa
}

Fecha de recepción: 20 de enero de 2020. Fecha de aceptación: 30 de junio de 2020.

https://doi.org/10.22201/iiec.20078951e.2021.Especial.69803

Resumen. La existencia de recursos públicos es esencial para la implementación de cualquier política pública. Cada gestión tiene el reto de encontrar el financiamiento para cumplir con la operación del gobierno y llevar a cabo los planes para el desarrollo de los países. Esta investigación contribuye al debate sobre el espacio fiscal y propone una metodología para su medición. A partir de la definición del espacio fiscal en términos del presupuesto disponible, se expone el caso mexicano y se abordan las opciones que existen para su ampliación a nivel federal.

Palabras clave: espacio fiscal; presupuesto público; política fiscal; financiamiento gubernamental; sostenibilidad financiera; recursos financieros no comprometidos.

Clasificación JEL: H61; H62; H63; O23.

\section{Measuring fiscal space in Mexico}

Abstract. The implementation of public policy depends on the availability of public resources. Governments, therefore, face the challenge of finding the funds necessary to execute national development plans. The present article contributes to the debate on fiscal space and proposes a methodology for its measurement. Based on the definition of fiscal space in terms of available budget, this article examines the Mexican case and discusses the existing options for its expansion at the federal level.

Key Words: fiscal space; public budget; fiscal policy; government financing; financial sustainability; non-committed financial resources.

${ }^{a}$ Escuela de Gobierno y Transformación Pública, Tecnológico de Monterrey, México. Correos electrónicos: hjvp@itesm.mx y A00777903@itesm.mx, respectivamente. 


\section{INTRODUCCIÓN}

Uno de los principales problemas para la aplicación de políticas públicas, en favor del desarrollo, es el insuficiente financiamiento gubernamental. En México, como en la mayoría de los países en desarrollo, los recursos públicos de libre disposición son restringidos en el corto plazo, lo que incentiva a los tomadores de decisiones a explorar estrategias para costear las políticas públicas a implementar en su gestión. Ros $(2008,2013,2015$ y 2019) puso en evidencia que para detonar el crecimiento económico en México es necesario orientar el espacio fiscal a factores de capital, como la educación, la salud e infraestructura en las regiones más desfavorecidas de los países.

El propósito de esta investigación es contribuir al debate sobre el concepto de espacio fiscal y proponer una metodología para su cuantificación en el corto, mediano y largo plazo. Mediante la revisión de literatura y la metodología planteada se responde a la pregunta: ¿qué es y cómo se mide el espacio fiscal? Con la aplicación de la metodología, propuesta al caso mexicano, se muestra la evolución del espacio fiscal en el país y se abordan las posibles estrategias que existen para su modificación a nivel federal.

Dado que no existe un consenso sobre el concepto de espacio fiscal, esta investigación abona al debate público en tres ámbitos: primero se expone una revisión de literatura para definir el espacio fiscal. Para ello, se confrontan tres perspectivas que refieren a este concepto: las que delimitan el espacio fiscal en función del endeudamiento; aquellas que aluden a la sostenibilidad de largo plazo; y las que utilizan la priorización del gasto público como medida del espacio fiscal. Como otra aportación se construye una definición del espacio fiscal a partir del presupuesto disponible en determinado punto del tiempo. Segundo, se propone una metodología para cuantificar el espacio fiscal. A diferencia de las aproximaciones existentes, el método aquí expuesto se propone sea asequible a cualquier disciplina, requiere de información puntual en materia fiscal, por lo que los insumos para su estimación lo hacen viable y robusto para la toma de decisiones en el corto plazo y es complementario a otras mediciones del espacio fiscal. Tercero, se realiza una aplicación de la metodología propuesta en este documento para el caso mexicano. Con las bases de datos sobre ingresos, gastos y deuda publicados por la Secretaría de Hacienda y Crédito Público (SHCP) anualmente, se estima el espacio fiscal disponible para el gobierno federal del 2013 al 2018, y queda para futuras administraciones la posibilidad de medir el espacio fiscal en años venideros. Adicionalmente, se exponen las posibles estrategias para la ampliación de recursos, con las cuales se podrá habilitar el financiamiento necesario para implementar políticas públicas. 
Después de la medición del espacio fiscal y ante mayores necesidades de financiamiento, esta investigación expone que las vías para la ampliación de recursos públicos de libre disposición son: la ayuda exterior, el incremento de impuestos domésticos, el uso eficiente o la redefinición del gasto público y el endeudamiento (Development Committee, 2006). Cada una de estas alternativas tendrá sus propias implicaciones para el caso mexicano, las cuales se exhiben al final de la investigación.

\section{EL CONCEPTO DE ESPACIO FISCAL}

El espacio fiscal es un tema de discusión vigente y su definición aún no termina de ser consensuada. Las principales propuestas, que anteceden esta investigación, se pueden clasificar en tres: las que lo analizan desde una perspectiva de sostenibilidad fiscal; aquellas que refieren a los niveles de endeudamiento y a la capacidad de pago de la deuda contratada; y quienes se enfocan en dirigir recursos a los objetivos de política pública para el desarrollo. En la metodología que se propone más adelante, se traza una nueva vertiente con una visión que reconoce los compromisos ineludibles en el presupuesto público para la medición del espacio fiscal.

Los distintos enfoques del espacio fiscal permiten que las metodologías para la medición y los resultados diverjan entre sí. Algunos de ellos enfatizan la necesidad de reconocer las características específicas de cada país, mientras que otros invitan al análisis comparado entre países. Con la metodología propuesta en esta investigación se favorece el análisis específico para cada país, con la posibilidad de ampliar el análisis hacia una perspectiva comparada.

\section{El espacio fiscal a través de la sostenibilidad de largo plazo}

Para Heller (2005) y Celasun et al. (2015), el espacio fiscal es el margen que existe dentro del presupuesto público que proporciona recursos para la aplicación de política pública; lo cual no debe comprometer la sostenibilidad financiera de largo plazo, ni la estabilidad de la economía. Ésta ha sido una de las definiciones de espacio fiscal más aceptadas, en ella se asume que existe sostenibilidad fiscal siempre que haya suficiencia fiscal.

Esta corriente se enfoca en las características específicas de los países para los cuales se realice la medición, y considera las limitaciones de cambios fiscales en el corto, mediano y largo plazo. Las propuestas metodológicas de Heller (2005) y Celasun et al. (2015) hacen énfasis en el largo plazo, en el cual se 
deben observar los indicadores sobre expectativas macroeconómicas, fiscales y de ayuda exterior en los años futuros para preservar la sostenibilidad fiscal.

El término de sostenibilidad fiscal es un debate por sí mismo, pues existen distintas maneras de entenderla, entre las más relevantes están: las relaciones entre la política fiscal y el crecimiento económico; las relaciones entre la inversión social y el crecimiento; o las condiciones en las cuales el crecimiento se traduce en un desarrollo sustentable en los países (International Labour Office, 2012). Por lo tanto, medir el espacio fiscal en función de la sostenibilidad de largo plazo, puede resultar ambiguo, ya que dependerá de la definición de sostenibilidad que se adopte.

\section{El espacio fiscal a través del endeudamiento}

Por otra parte, para autores como Ghosh et al. (2013), Ostry et al. (2010) y Pommier (2015), el espacio fiscal depende de la capacidad de contraer pasivos en cada país. En este caso, las relaciones de deuda sobre el Producto Interno Bruto (PIB) son utilizadas para medir la sostenibilidad fiscal de largo plazo. Para estos autores, la diferencia entre el nivel de deuda con la capacidad máxima de endeudamiento cuantifica el espacio fiscal.

En tanto, Aizenman y Jinjarak (2010) miden el espacio fiscal en función de los años fiscales que necesitaría un país para pagar la deuda pública. Bajo esta perspectiva, la capacidad recaudatoria de los países es un factor importante, así como las fluctuaciones de los ingresos públicos debido a los ciclos económicos.

Esta corriente prioriza el pago de las obligaciones financieras frente a las necesidades de política para el desarrollo o crecimiento económico, así como la aplicación de nuevos programas en el corto plazo, suponiendo que habrá sostenibilidad fiscal. Su medición del espacio fiscal permite la comparación entre países, sin hacer distinción en las características específicas de cada uno y sus necesidades de política para el desarrollo. Las estimaciones que se realizan son de predicción y funciones de reacción, las cuales restringen su aplicabilidad en países donde el desglose de la información fiscal de largo plazo es limitado.

\section{Espacio fiscal para la agenda del desarrollo}

Autores como Roy et al. (2007), Aguzzoni (2011) y Lofgren (2013) cuestionan la finalidad del espacio fiscal. Para ellos, la métrica principal es la existencia de 
recursos para impulsar la agenda en favor del desarrollo de los países, principalmente, en términos de educación, salud e infraestructura. Estos autores, así como Perotti (2007) y Heller (2005), coinciden en que para ampliar recursos para algún fin es necesario recortar en otros sectores, o bien obtener recursos de otros lados que terminarán afectando de alguna manera a la población.

En esta corriente del espacio fiscal se supone que la priorización del gasto puede ser cambiada sustancialmente en el corto plazo, sin reconocer las limitantes legales y de economía política que existen en los países. De modo que existirá espacio fiscal siempre que el gasto público y los recursos adquiridos por mayores ingresos, endeudamiento o ayuda internacional sean dirigidos a sectores que impulsen el desarrollo, tales como educación, salud, alimentación, vivienda, entre los más relevantes.

Para la medición del espacio fiscal bajo la corriente de priorización de la agenda para el desarrollo se requiere de un análisis específico en cada país. Debido a que las necesidades en cada nación son diferentes, no es posible realizar una comparación entre ellas. Por lo tanto, para cuantificar el espacio fiscal, bajo esta perspectiva, se requieren de dos procesos: conocer la agenda necesaria para el desarrollo, y luego examinar la posibilidad de dirigir recursos a estos fines.

\section{Espacio fiscal en el presupuesto disponible}

Las corrientes sobre espacio fiscal expuestas anteriormente definen y miden de distintas maneras este complejo concepto. Por un lado, la corriente sobre sostenibilidad fiscal resalta la importancia de mantener la disponibilidad de recursos en el largo plazo. Por otro, la corriente que da importancia al endeudamiento abre la posibilidad de tener mayores recursos en función de las capacidades de pago de los países. Además, la corriente que pone al centro de la discusión a la agenda del desarrollo invita a pensar en los propósitos para los cuales se requiere el espacio fiscal. Estas distintas maneras de entender el espacio fiscal permiten formular la corriente del espacio fiscal que aquí se propone: la del presupuesto disponible. La cual no pretende reemplazar las mediciones existentes de espacio fiscal, sino ser complementaria para la toma de decisiones en el corto plazo.

En esta investigación se define al espacio fiscal como los recursos financieros no comprometidos en el presupuesto anual y que pueden ser utilizados para ejercer política pública de coyuntura o de nueva creación, así como los proyectos que abonan al desarrollo de los países. De este modo, se concibe al 
espacio fiscal como un conjunto de recursos financieros que pueden cambiar en el corto (un año o menos), mediano (entre uno y tres ańos) y largo plazo (tres años o más). ${ }^{1}$

A diferencia de las tres corrientes expuestas anteriormente, el concepto que aquí se presenta reconoce que el espacio fiscal puede cambiar a lo largo del tiempo, ya que depende de las políticas públicas implementadas en años anteriores y otras políticas vigentes al momento de la medición. Se advierte que las políticas públicas de hoy pueden tener efectos importantes en el espacio fiscal disponible en el futuro, a través del crecimiento económico que generen, afectando a las generaciones que aún no han nacido, así como a la sostenibilidad fiscal. ${ }^{2}$

Para la cuantificación del espacio fiscal, las corrientes que anteceden a esta nueva perspectiva requieren de series de tiempo de largo plazo o que se enfocan en un conjunto de indicadores macro y microeconómicos, tales como el crecimiento económico esperado, las relaciones de deuda y producción, así como las estructuras de ingresos y gastos en los hogares. Obtener una serie lo suficientemente robusta de todos los indicadores, en algunos países puede ser una limitante. Además, dejan de lado la necesidad de los agentes políticos de recursos en el corto plazo, y no hacen explícitas las restricciones legales para la construcción del espacio fiscal de manera inmediata.

Lo que aquí se propone parte del análisis de la estructura presupuestal del país de interés en determinado periodo. Por lo que, no resultan necesarias largas series de tiempo para su medición. Además, con la aplicación de la metodología, explicada en la siguiente sección, puede realizarse un análisis comparado entre países y distintos puntos en el tiempo. Esta propuesta, al tener un enfoque de corto plazo, considera los intereses políticos que existen por la disponibilidad de recursos, así como las limitantes en modificar los presupuestos y las políticas tributarias debido a candados políticos y legales. También permite incorporar consideraciones institucionales de manera explícita, lo cual resulta complicado en los otros enfoques presentados.

1 Para el caso mexicano se consideran tres años, ya que corresponde con el cambio de legisladores en el Congreso.

2 Elemento central en el pensamiento de Ros, quien defendía la idea de que el gasto público, diferenciando el gasto corriente del gasto en formación de capital físico y humano, así como por regiones, repercutía de manera endógena en el crecimiento y, por ende, en la capacidad financiera del gobierno (Ros, 2013 y 2015). 


\section{METODOLOGÍA PARA MEDIR EL ESPACIO FISCAL}

Para delimitar el espacio fiscal intervienen distintos actores que se establecen a través de las instituciones que interactúan en el sistema fiscal. Los principales son el conductor de la política fiscal, que en el caso mexicano es la SHCP, ya que administra los recursos entre programas y proyectos que el gobierno ha de implementar; los ciudadanos quienes pagan impuestos y a su vez demandan bienes y servicios públicos; los legisladores quienes establecen la regulación correspondiente; las empresas que además de pagar impuestos y demandar bienes y servicios públicos también inciden (como las personas) en el desarrollo del país, entre otros.

La metodología propuesta parte del corto plazo, es de rápida aplicación, y consistente en resultados con otras metodologías (FMI, 2018), lo que permite que los hacedores de política pública tengan herramientas técnicas para conocer la disponibilidad de los recursos públicos en un año determinado. Para su desarrollo, se requiere el conocimiento de estructuras presupuestales y legislaciones reguladoras, sin ahondar en las relaciones que se refieren en las estimaciones de equilibrio general que modelan el sistema fiscal (Llop y Flores, 2005; Núñez-Rodríguez, 2015; Pedauga et al., 2012; Sobarzo, 2009). En este sentido, el trabajo de Ros (2008, 2013, 2015 y 2019) presenta evidencia sobre la necesidad de orientar el espacio fiscal disponible en factores de capital, especialmente de educación, salud e infraestructura en las regiones más desfavorecidas para abonar al crecimiento económico de los países.

En los presupuestos públicos existen conceptos que son ineludibles en el mediano y largo plazo (Banco Mundial, 2016; Cetrángolo et al., 2010). Dichas erogaciones dependen de las limitantes legales y políticas que se hayan impuesto a los recursos públicos con anterioridad. Lo que no implica que sean rígidos perpetuamente, sino que se requieren cambios estructurales y voluntad política para su liberación.

A diferencia de lo planteado en el resto de la literatura sobre espacio fiscal, donde se proponen una serie de indicadores al respecto, entre ellos las aproximaciones de endeudamiento que requieren largas series de tiempo (FMI, 2017; Kose et al., 2017), esta metodología permite tener una estimación puntual del espacio fiscal disponible, la cual puede ser aplicada en distintos periodos. De tal modo que, al contrastar los gastos que son ineludibles con los ingresos públicos de determinado periodo, se encuentra el espacio fiscal del año en cuestión.

Espacio_fiscal $_{i}=$ Ingresos_públicos $_{i}-$ Gastos_ineludibles $_{i}$ 
Los gastos ineludibles en el largo plazo son los recursos que se encuentran comprometidos por obligaciones de pago con terceros y por cuestiones legales. Se les clasifica de esta manera porque su liberación, bajo el supuesto que se cambia la ley o los compromisos, será posible en un periodo de tres ańos o más, lo que se vincula a los cambios de legislatura en los congresos y la voluntad política existente.

Por otro lado, existen programas puestos en marcha a través de reglas de operación y que tienen una población beneficiaria definida que dependen directamente del apoyo recibido en ańos previos. Estos apoyos, aunque son modificables, deberán someterse a revisión para compensar de alguna manera los beneficios recibidos por la población, de tal manera que no haya mayor afectación política. Por lo tanto, serán ineludibles en el mediano plazo.

Existen otros gastos que, al no estar comprometidos por una ley, generalmente no implican un servicio básico directo a la población y sus recursos pueden ser modificados en el corto plazo, por lo que forman parte del espacio fiscal disponible. De tal modo que, el diferencial entre los ingresos públicos totales, sin considerar endeudamiento y los gastos ineludibles del mediano y largo plazo dan como resultado el espacio fiscal disponible para la coyuntura.

\section{MEDICIÓN DEL ESPACIO FISCAL EN MÉXICO}

México, como muchos países, ha pasado por una transición política y económica. El resultado de la elección federal de 2018 fue un parteaguas para la política fiscal en el país. Tras los cambios de gobierno, los nuevos administradores públicos regularmente desean aplicar políticas públicas y programas que diferencien a su gestión, dejando atrás los programas emblemáticos de gestiones anteriores. Ante la necesidad de recursos para la implementación de los nuevos programas prioritarios es necesario conocer, medir y explorar el espacio fiscal disponible en el corto, mediano y largo plazo.

Para la estimación del espacio fiscal en México se utiliza la metodología expuesta en el apartado anterior, con datos publicados por la SHCP sobre ingresos tributarios y no tributarios, así como de la Cuenta de la Hacienda Pública Federal. Se analiza el periodo del 2013 al 2018 de forma anual.

Tras analizar la estructura del presupuesto federal se encuentra que los gastos ineludibles, que intervienen en la estimación del espacio fiscal, son identificables en el presupuesto de egresos de la Federación, así como en la cuenta pública de la hacienda federal de cada año. Para ello se recurre a la clasificación 
por "modalidad" de los gastos presupuestarios, disponibles tanto en las cuentas públicas como en el presupuesto en los años de estudio.

Los programas ineludibles, que pueden cambiar en el largo plazo, se identifican bajo las modalidades de: Prestación de servicios públicos, donde se incluye todo el pago a la nómina de profesores y personal médico; Pensiones y Jubilaciones; Gasto federalizado; Aportaciones a la seguridad social; Desastres naturales; Participaciones a las entidades federativas y municipios; Costo financiero; Deuda o apoyos a deudores y ahorradores de la banca; y los Adeudos de ejercicios fiscales anteriores. Cabe mencionar que estos programas no forman parte del gasto corriente estructural. ${ }^{3}$

Aquellos programas que pueden ser modificados en el mediano plazo se identifican en las siguientes modalidades de programas: Programas sujetos a reglas de operación; Otros subsidios; Específicos; Provisión de bienes; Funciones de las Fuerzas Armadas; y las Obligaciones de cumplimiento de resolución jurisdiccional. El resto de las modalidades no mencionadas refieren a programas que potencialmente pueden modificarse en el corto plazo y, por lo tanto, ser parte del espacio fiscal disponible.

\section{RESULTADOS SOBRE EL ESPACIO FISCAL EN MÉXICO}

$\mathrm{Al}$ identificar los programas presupuestarios según la modalidad dentro de las cuentas públicas se encuentra que, en promedio del 2013 al 2018, 63.7\% del total del gasto federal ha estado comprometido en el largo plazo (véase cuadro 1). Entre los principales rubros ineludibles se encuentran: el gasto dirigido a las entidades federativas, que en promedio del periodo representa $25.4 \%$ del gasto neto total; el pago de pensiones y jubilaciones con $12.1 \%$; así como el costo financiero de la deuda con 7.3\%. Estos gastos podrían ser modificados de manera significativa al haber una reforma a la Ley de Coordinación Fiscal o de las leyes que regulan el sistema de pensiones en México.

Por otro lado, los programas que pueden ser sujetos a revisión y, por lo tanto, ser modificados en un periodo de entre uno y tres años han representado,

3 El artículo 2 XXIVBIS de la Ley Federal de Presupuesto y Responsabilidad Hacendaria define al gasto corriente estructural como el gasto neto total, sin considerar los gastos por concepto de costo financiero, participaciones a las entidades federativas, a los municipios y a las demarcaciones territoriales de la Ciudad de México, adeudos de ejercicios fiscales anteriores, combustibles utilizados para la generación de electricidad, pago de pensiones y jubilaciones del sector público. 
en promedio del 2013 al 2018, 21.9\% del total del gasto público. Este tipo de programas disminuyeron su participación en el gasto público total a partir de la restructuración del presupuesto a nivel federal ocurrida en 2015. Los principales representantes de esta categoría se refieren a los programas que otorgan algún tipo de subsidio a las personas físicas y morales a través de programas sujetos a reglas de operación, programas específicos y otros de subsidios. Los cuales en promedio han participado del presupuesto con un 5.9, 3.7 y $6.7 \%$, respectivamente.

El presupuesto que puede ser modificable en el corto plazo y, por tanto, ser parte del espacio fiscal disponible de forma anual, en promedio ha representado $14.4 \%$, considerando el monto asignado a proyectos de inversión. De no considerarse los recursos para proyectos inversión, pues su disminución afectaría el crecimiento (Ros, 2015), el espacio fiscal disponible será de 5.9\% del total del gasto público, durante el periodo de estudio. Los gastos de esta categoría incluyen los programas de Apoyo al proceso presupuestario y para mejorar la eficiencia institucional; y Planeación, seguimiento y evaluación de políticas públicas. Ambas modalidades de programas pertenecen a organismos que son autónomos o descentralizados al gobierno central y aunque pueden ser modificables en un año o menos, esta decisión no depende directamente del Poder Ejecutivo federal.

En el cuadro 1 se muestra la distribución del gasto público total, según modalidad de programa y se identifican según el periodo de posible modificación. Los resultados reflejan que los gastos ineludibles son superiores al 60\% para todos los años de estudio y que se han incrementado después de algún ajuste fiscal, como la Reforma Hacendaria de 2014 y la reestructura del gasto público reflejada en 2016. En contraste, la proporción del gasto destinada a los programas susceptibles de cambio en el corto plazo y que abonan al espacio fiscal ha disminuido a través de los años.

Por su parte, los ingresos del sector público no han sido de la misma magnitud que los gastos del gobierno. Del 2013 al 2019, los egresos totales superaron los ingresos totales, sin considerar el endeudamiento adquirido. En 2014 y 2015 la brecha fue de alrededor del 16\% de los ingresos totales, lo que dio pie a la reestructura del gasto. Para 2018 este diferencial se redujo a 5.2\% debido a un incremento en la recaudación y la contención del gasto público federal. El diferencial necesario para cubrir las erogaciones públicas se ha adquirido a través del endeudamiento. En el largo plazo, este déficit puede ejercer presión sobre el costo financiero de la deuda que deberá pagarse en años venideros y con ello implicar una contracción del espacio fiscal. 
Cuadro 1. Clasificación del gasto público según modalidad

\begin{tabular}{|c|c|c|c|c|c|c|c|}
\hline $\begin{array}{l}\text { Tipos de programas } \\
\text { presupuestarios }\end{array}$ & $\begin{array}{c}2013 \\
(\%)\end{array}$ & $\begin{array}{c}2014 \\
(\%)\end{array}$ & $\begin{array}{c}2015 \\
(\%)\end{array}$ & $\begin{array}{c}2016 \\
(\%)\end{array}$ & $\begin{array}{c}2017 \\
(\%)\end{array}$ & $\begin{array}{c}2018 \\
(\%)\end{array}$ & $\begin{array}{l}\text { Promedio } \\
(\%)\end{array}$ \\
\hline $\begin{array}{l}\text { Programas ineludibles ( I+ II) } \\
\text {-largo plazo- }\end{array}$ & 62.3 & 61.4 & 62.4 & 60.4 & 66.6 & 69.1 & 63.7 \\
\hline I. Gasto Programable & 42.8 & 41.7 & 42.5 & 40.3 & 43.7 & 44.8 & 42.6 \\
\hline Prestación de servicios públicos & 19.4 & 18.1 & 18.4 & 16.8 & 18.3 & 18.9 & 18.3 \\
\hline Pensiones y Jubilaciones* & 10.9 & 11.3 & 11.8 & 11.8 & 13.0 & 13.7 & 12.1 \\
\hline Gasto federalizado & 12.4 & 12.2 & 12.3 & 11.6 & 12.3 & 12.1 & 12.1 \\
\hline Aportaciones a la seguridad social* & 0.1 & 0.1 & 0.1 & 0.1 & 0.1 & 0.1 & 0.1 \\
\hline II. Gasto No Programable & 19.5 & 19.8 & 19.9 & 20.1 & 22.9 & 24.3 & 21.1 \\
\hline $\begin{array}{l}\text { Participaciones a las entidades } \\
\text { federativas y municipios }\end{array}$ & 12.5 & 12.7 & 12.7 & 12.7 & 14.4 & 14.7 & 13.3 \\
\hline $\begin{array}{l}\text { Costo financiero, deuda o apoyos a } \\
\text { deudores y ahorradores de la banca }\end{array}$ & 6.6 & 6.6 & 6.6 & 7.0 & 8.2 & 8.5 & 7.3 \\
\hline $\begin{array}{l}\text { Adeudos de Ejercicios Fiscales } \\
\text { Anteriores }\end{array}$ & 0.4 & 0.5 & 0.5 & 0.4 & 0.4 & 1.1 & 0.5 \\
\hline $\begin{array}{l}\text { Programas sujetos a revisión } \\
\text {-mediano plazo- }\end{array}$ & 22.7 & 22.8 & 23.2 & 20.4 & 22.6 & 19.5 & 21.9 \\
\hline Sujetos a reglas de operación & 6.3 & 7.1 & 6.3 & 5.8 & 4.9 & 4.8 & 5.9 \\
\hline Otros subsidios & 6.7 & 6.4 & 7.3 & 6.5 & 6.9 & 6.5 & 6.7 \\
\hline Específicos* & 3.5 & 4.6 & 3.9 & 3.1 & 3.6 & 3.6 & 3.7 \\
\hline Provisión de bienes públicos & 3.2 & 2.1 & 2.9 & 1.4 & 1.4 & 1.3 & 2.1 \\
\hline Funciones de las Fuerzas Armadas & 1.8 & 1.8 & 1.8 & 1.7 & 1.8 & 1.8 & 1.8 \\
\hline Desastres Naturales & 0.3 & 0.3 & 0.5 & 0.3 & 0.2 & 0.6 & 0.4 \\
\hline $\begin{array}{l}\text { Obligaciones de cumplimiento de } \\
\text { resolución jurisdiccional }\end{array}$ & 0.0 & 0.0 & 0.0 & 0.0 & 0.0 & 0.0 & 0.0 \\
\hline $\begin{array}{l}\text { Aportaciones a fondos de } \\
\text { estabilización }\end{array}$ & 0.9 & 0.4 & 0.4 & 1.5 & 3.8 & 0.9 & 1.3 \\
\hline Otros programas -corto plazo- & 15.0 & 15.8 & 14.4 & 19.1 & 10.8 & 11.4 & 14.4 \\
\hline $\begin{array}{l}\text { Apoyo a la función pública y } \\
\text { al mejoramiento de la gestión }\end{array}$ & 0.1 & 0.1 & 0.1 & 0.1 & 0.1 & 0.1 & 0.1 \\
\hline
\end{tabular}


Cuadro 1. Clasificación del gasto público según modalidad (continuación)

\begin{tabular}{|c|c|c|c|c|c|c|c|}
\hline $\begin{array}{l}\text { Tipos de programas } \\
\text { presupuestarios }\end{array}$ & $\begin{array}{c}2013 \\
(\%)\end{array}$ & $\begin{array}{c}2014 \\
(\%)\end{array}$ & $\begin{array}{c}2015 \\
(\%)\end{array}$ & $\begin{array}{c}2016 \\
(\%)\end{array}$ & $\begin{array}{r}2017 \\
(\%)\end{array}$ & $\begin{array}{c}2018 \\
(\%)\end{array}$ & $\begin{array}{l}\text { Promedio } \\
(\%)\end{array}$ \\
\hline $\begin{array}{l}\text { Apoyo al proceso presupuestario } \\
\text { y para mejorar la eficiencia } \\
\text { institucional }\end{array}$ & 3.0 & 3.0 & 3.0 & 2.8 & 3.0 & 3.2 & 3.0 \\
\hline Operaciones ajenas & -0.5 & -0.2 & -0.1 & -0.3 & -0.2 & -0.2 & -0.3 \\
\hline $\begin{array}{l}\text { Planeación, seguimiento y } \\
\text { evaluación de políticas públicas }\end{array}$ & 0.9 & 1.1 & 1.3 & 7.0 & 1.1 & 1.1 & 2.1 \\
\hline Promoción y fomento & 0.3 & 0.3 & 0.4 & 0.3 & 0.3 & 0.3 & 0.3 \\
\hline Proyectos de inversión & 10.6 & 10.8 & 9.0 & 8.6 & 6.0 & 6.4 & 8.6 \\
\hline Regulación y supervisión & 0.6 & 0.6 & 0.6 & 0.5 & 0.5 & 0.5 & 0.6 \\
\hline Gasto neto de transferencias & 100.0 & 100.0 & 100.0 & 100.0 & 100.0 & 100.0 & 100.0 \\
\hline $\begin{array}{l}\text { Menos cuotas al ISSSTE+intereses } \\
\text { compensados (CP) }\end{array}$ & 1.1 & 0.9 & 0.7 & 1.3 & 1.7 & 2.3 & 1.3 \\
\hline Gasto Neto Total & 98.9 & 99.1 & 99.3 & 98.7 & 98.3 & 97.7 & 98.7 \\
\hline
\end{tabular}

Nota: * se descuentan las transferencias realizadas al IMSS e ISSSTE por ramo 19.

Fuente: elaboración propia con datos de la SHCP (2013-2018). Cuenta de la Hacienda Pública Federal.

En los periodos en los que el gasto ineludible, más el asignado a los programas sujetos a revisión, se aproximó al total de los ingresos del sector público mexicano se promovieron acciones para buscar la ampliación del espacio fiscal. En 2014 se aprobó una Reforma Hacendaria que incrementó los recursos en años posteriores. En 2015 se comenzó la reestructura del gasto público que contuvo la expansión de los programas sujetos a revisión y de otros programas (véase gráfica 1).

A pesar de los esfuerzos realizados con la Reforma Hacendaria y la reestructura del gasto, al contrastar los ingresos públicos con los gastos ineludibles de largo plazo, se encuentra un espacio fiscal en constante contracción, exceptuando el espacio generado en 2016 por el remanente del Banco de México. Lo anterior se relaciona con los compromisos del gobierno mexicano de mantener un superávit primario, sin crear nuevos impuestos o alzas a las tasas tributarias federales. Al considerar la proporción del gasto ineludible y el asignado a los programas clasificados como sujetos a revisión, el espacio fiscal que tiene el país en 2018, para la realización de nuevas políticas públicas en 
Gráfica 1. Relaciones del espacio fiscal en México (millones de pesos corrientes)

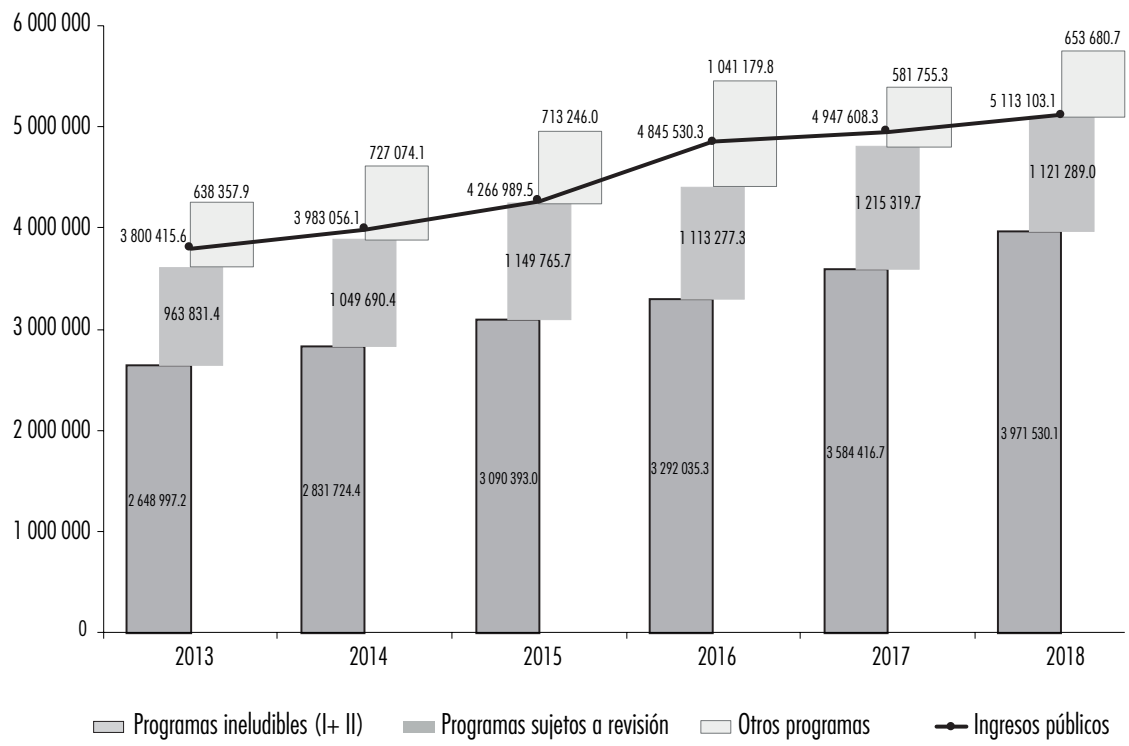

Fuente: elaboración propia con datos de la SHCP (2013-2019). Cuenta de la Hacienda Pública Federal.

Gráfica 2. El espacio fiscal en México 2013-2018 (porcentaje del PIB)

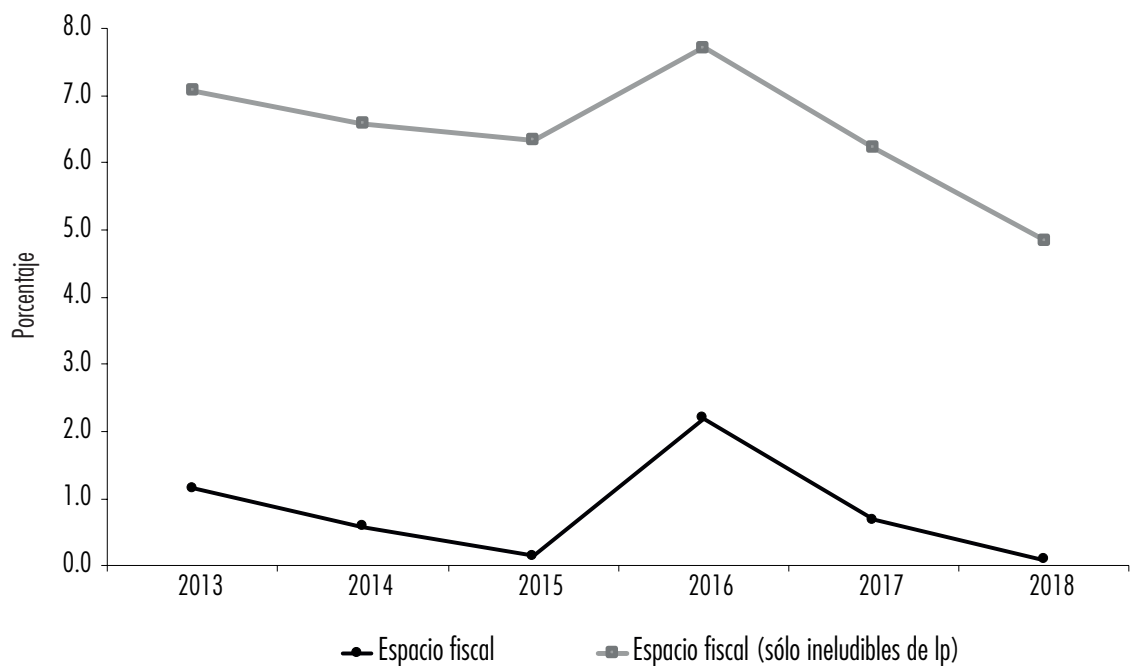

Fuente: elaboración propia con datos de la SHCP (2013-2018). Cuenta de la Hacienda Pública Federal. 
el corto plazo, es menor a un punto del PIB (véase gráfica 2). Una reestructuración del gasto de los programas sujetos a revisión en México pudiera liberar un espacio fiscal de 5 puntos PIB, manteniendo lo demás constante. Este resultado es consistente con lo presentado por el Fondo Monetario Internacional (FMI) en 2018, donde se clasifica a México con "algún espacio fiscal" derivado de las ineficiencias del gasto público, su limitada recaudación y la razón de deuda sobre PIB superior al 50\%.

Un espacio fiscal en contracción limita la posibilidad del gobierno en turno de financiar una nueva política pública que sea emblemática en su gestión y sostenerla a lo largo de un sexenio, sin lacerar las políticas existentes. Por lo tanto, resulta relevante analizar las alternativas que existen para ampliar el espacio fiscal.

\section{ESTRATEGIAS PARA AMPLIAR EL ESPACIO FISCAL}

En 2006, el Comité de Desarrollo del Banco Mundial y el FMI reconocieron cuatro acciones posibles para la movilización de recursos con el fin de ampliar el espacio fiscal. Y son la asistencia internacional para el desarrollo, el endeudamiento, la movilización de ingresos internos (principalmente fiscales), así como la priorización y eficiencia en el uso de los recursos públicos. Estas estrategias, consensuadas en el ámbito internacional, también son aplicables para el caso mexicano.

\section{Asistencia internacional}

Los flujos de efectivo provenientes del extranjero pueden, en el corto y mediano plazo, financiar algunos proyectos, con lo que se liberan recursos para otros fines. Empero, esta ayuda generalmente también representa costos de gestión y compromisos de financiamiento para proyectos o programas predeterminados. Además, para países de ingreso medio, como México, estos recursos no representan una parte significativa de su presupuesto. La ayuda internacional que ha recibido México, en la mayoría de los casos, va dirigida a proyectos específicos. Por lo tanto, las contribuciones que se reciben no impactan de manera directa al presupuesto de egresos de la Federación ni a la ampliación de recursos para política pública de coyuntura.

En 2011, México recibió US\$971 millones por concepto de ayuda internacional, siendo éste el máximo desde 2008 cuando se recibieron US\$149 
millones (Secretaría de Gobernación, 2014). En 2017, el país recibió US $\$ 737.5$ millones, superando la meta del Programa de Cooperación Internacional para el Desarrollo 2014-2018 (Secretaría de Relaciones Exteriores, 2018). Para 2018 se proyectó superar los US $\$ 418$ millones. Dado que los recursos recibidos fueron destinados a actividades específicas, como el terremoto del 2017, no forman parte del espacio fiscal disponible para implementar política pública de coyuntura de manera sostenida. Aunque la disponibilidad de estos recursos puede liberar presión para financiar algunos otros programas que representen menos del $1 \%$ del PIB, dada la volatilidad de su recepción, no pueden ser considerados para la aplicación de política pública de mediano o largo plazo.

\section{Endeudamiento}

El endeudamiento interno y externo es otra forma de ampliar el espacio fiscal. Para recurrir a esta fuente de recursos se debe considerar que todo endeudamiento debe ser pagado en algún punto del tiempo, afectando a generaciones presentes y futuras.

En México, los requerimientos financieros del sector público presupuestario, que reflejan el endeudamiento público presupuestario y no presupuestario, pueden derivarse de las siguientes vertientes: del balance tradicional; de los requerimientos financieros de Proyectos de Inversión de Infraestructura Productiva con Registro Diferido en el Gasto Público (PIDIREgas); de adecuaciones a los registros presupuestarios; de recursos para el Fondo Nacional de Infraestructura (FONADIN); del programa de apoyo a deudores y de requerimientos del Instituto para la Protección al Ahorro Bancario (IPAB); y de requerimientos de financieros de la banca de desarrollo y fondos de fomento que resultan de la ganancia o pérdida esperada del crédito otorgado por intermediación de los bancos de desarrollo y los fondos de fomento (Vázquez, 2019). Recurrir al endeudamiento como medida de ampliación fiscal tiene implicaciones de corto y largo plazo. Si bien puede aumentar los recursos en determinado punto del tiempo, de no ser utilizados para proyectos productivos, que generen mayores rendimientos que la tasa de interés a la que fueron adquiridos, en el mediano plazo el endeudamiento representará un costo mayor, incrementando los requerimientos financieros iniciales. 
Gráfica 3. Requerimientos financieros del sector público $2013-2018$ (porcentaje del PIB)

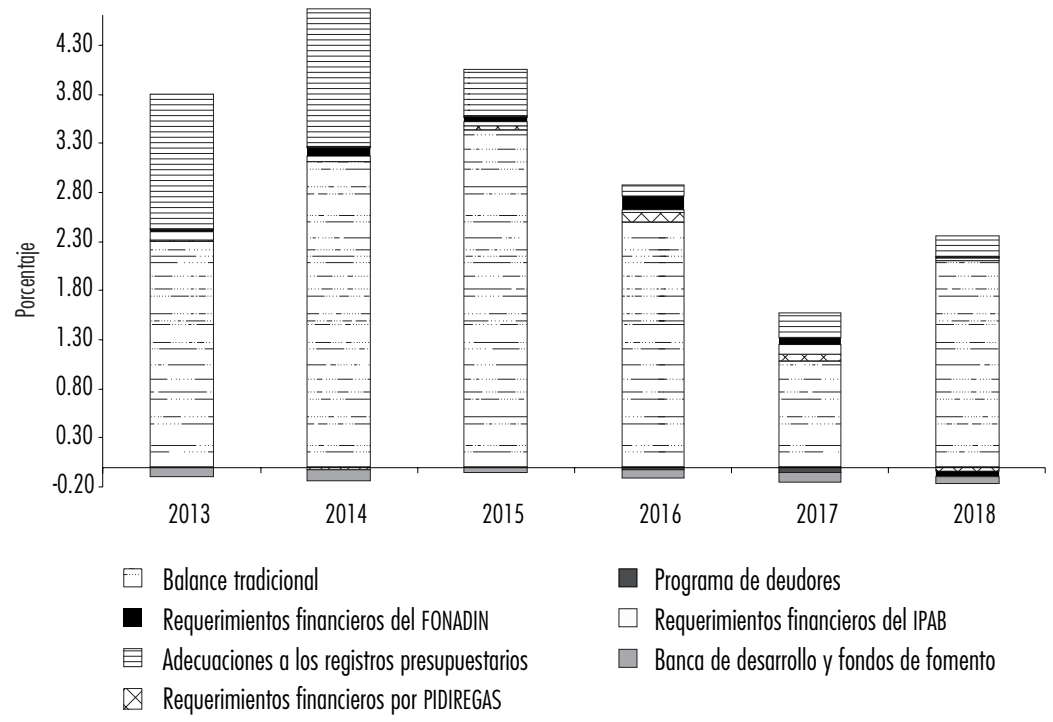

Fuente: elaboración propia con datos de la SHCP (2019). Estadísticas Oportunas. Requerimientos Financieros del Sector Público Federal por componente.

Del 2013 al 2018 los requerimientos financieros del sector público oscilaron entre 1.1 y $4.5 \%$ del PIB. La mayor proporción se deriva de los requerimientos del balance tradicional, seguido de las adecuaciones a los registros presupuestarios, que emanan de los requerimientos por la inflación en los instrumentos financieros dentro del presupuesto (véase gráfica 3). Estos requerimientos se han utilizado en mayor medida para cubrir la operación del gobierno federal, pues representan en promedio $46 \%$ del espacio fiscal en su definición más laxa. Lo anterior pone como evidencia el uso del endeudamiento como medida de ampliación del espacio fiscal en el país.

\section{Movilización de ingresos internos}

El incremento en la recaudación es la forma directa de ampliar el espacio fiscal. Con mayores tasas impositivas, menores exenciones y bases tributarias más amplias se pueden obtener recursos para el financiamiento de políticas públicas. Sin embargo, esta medida podría incrementar la pobreza y tener efectos adversos en el crecimiento económico (Estevão, 2019). Por otro lado, 
el costo político y social de aumentar la recaudación a través del incremento de tasas no es siempre viable.

Además del incremento de tasas y bases gravables, existen otras maneras que pueden incidir en la recaudación como la reducción de la elusión y la evasión. Alm (2012) sugiere acciones en contra del comportamiento ilegal, como mayores y mejores auditorías, así como castigos más severos.

Asimismo, son necesarias mejoras a la Administración Tributaria respecto del servicio que se le ofrece al contribuyente. Se requiere mejorar la asistencia técnica y simplificar los procesos de pago, ya que sistemas tributarios complejos dan oportunidad a la evasión y la corrupción. Según Awasthi y Bayraktar (2014), una reducción del $10 \%$ en el número de pagos y en el tiempo necesario para cumplir con los requisitos tributarios disminuiría la corrupción tributaria en $9.6 \%$, y representaría un aumento en la recaudación.

Incorporar la economía digital también contribuye al incremento de los ingresos. Medidas como permitir el pago de impuestos de manera electrónica es un primer avance. Una vez que se cuenta con la infraestructura básica para la economía digital se pueden implementar otras que llevarán a una la recaudación más eficiente tales como: la identificación digital, las finanzas digitales, el seguimiento en línea de facturas y ventas, las declaraciones de impuestos que se llenan automáticamente, entre otras (Estevão, 2019). Todas ellas vinculadas a garantizar que no exista una doble tributación y un nivel mínimo de aportación (Rafiq, 2019).

Estas medidas deben ser acompañadas de un cambio de cultura en el pago de impuestos, donde el ciudadano es consciente de la relación entre pago de impuestos y provisión de servicios públicos. Crear confianza en el ciudadano y crear un círculo virtuoso entre el pago de impuestos y los servicios públicos reforzará la visión ética del pago de ellos (Estevão, 2019).

Aunque lo deseable es una Reforma Fiscal que incremente los recursos públicos con bases gravables más amplias y tasas progresivas, bien puede tomar varios ańos su implementación. Elementos como la formalidad, evasión y elusión son determinantes para el éxito de cualquier reforma, además de explorar nuevas fuentes de financiamiento como los impuestos al carbono y al capital, por mencionar algunos. Esta reforma permitiría financiar grandes proyectos en favor del crecimiento económico sin provocar una mayor desigualdad (Ros, 2014).

México es el país con menor recaudación tributaria entre los países de la Organización para la Cooperación y el Desarrollo Económicos (OCDE). En 2017 ingresó $16.1 \%$ del PIB por ingresos tributarios (véase gráfica 4). Los principales conceptos por los que se recauda a nivel federal son: 
- El impuesto al valor agregado (IVA) es un impuesto indirecto al consumo, que grava el valor añadido en cada etapa de la producción. El gravamen general es del $16 \%$, pero puede variar a tasa 0 o exento, según el tipo de bien. En 2018 este impuesto representó 30\% del total de los impuestos tributarios y representó $3.9 \%$ del PIB.

- El impuesto sobre la renta (ISR) grava los ingresos de nacionales y extranjeros que se produzcan en el país. La tasa depende del nivel de ingresos de las personas físicas o morales. En 2018 este impuesto representó 55\% de los impuestos tributarios y representa $7.1 \%$ del PIB.

- El impuesto especial sobre producción y servicios (IEPS) se aplica a la producción, enajenación e importación de ciertos bienes y servicios establecidos en la Ley del IEPs. La principal recaudación por este concepto recae en las gasolinas y el diésel, el cual equivale al $6 \%$ de la tributación. Existen IEPS a otros bienes y servicios, como bebidas azucaradas, alcohol, tabaco, entre otros; los cuales suman 5\% del ingreso. La percepción total por IEPS en 2018 fue del $1.5 \%$ del PIB.

- Existen otros impuestos como los derivados de exportaciones, importaciones, automóviles, entre otros que suman $4 \%$ de la recaudación tributaria y equivalen al $0.5 \%$ del PIB.

Gráfica 4. Ingresos tributarios en México 2013-2018 (porcentaje del PIB)

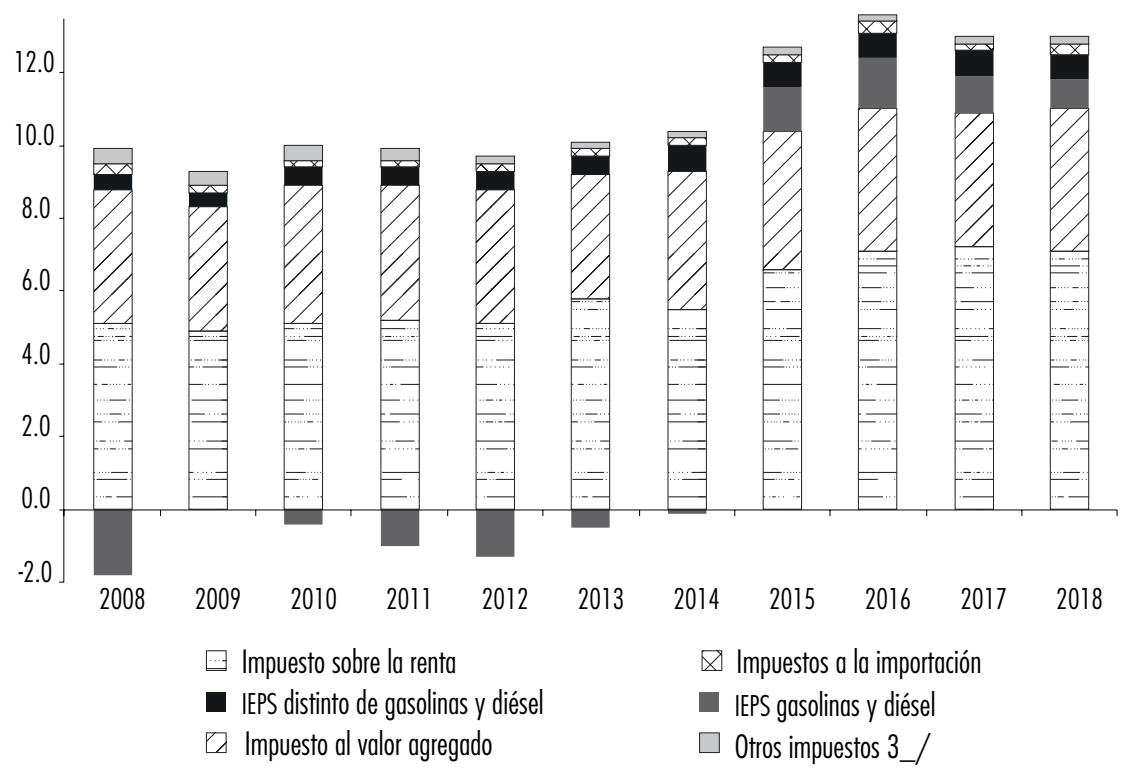

Fuente: elaboración propia con datos de la SHCP (2019). Estadísticas Oportunas. Ingresos presupuestarios del Sector Público. 
En la última década se implementaron diversas medidas en materia tributaria en México. En 2008 se redujo la tasa del impuesto a las ganancias corporativas. En 2009 disminuyó el impuesto a los activos, y se introdujo un nuevo impuesto de retención sobre los intereses de depósitos en efectivo. En 2010 se introdujeron sistemas de pago electrónico para impuestos sobre la nómina, la propiedad y la seguridad social. En 2011 se aumentaron varias tasas impositivas, incluido el impuesto a las ganancias corporativas y la tasa de depósitos en efectivo; se dieron más opciones para el pago en línea y otras soluciones tecnológicas para la administración contable. En 2012 se redujeron los requisitos para la presentación de impuestos por parte de las empresas, además de eliminar la declaración anual del IVA. Sin embargo, estos cambios no tuvieron efectos mayores al $1.3 \%$ del рів.

En 2014, México tuvo la mayor Reforma Fiscal de la última década. Los principales cambios, aprobados por el Legislativo, fueron: la homologación del IVA en la frontera, que paso del 11 al 16\%; una mayor progresividad del ISR; la eliminación del Impuesto Empresarial a Tasa Única (IETU) y el Impuesto a los Depósitos en Efectivo (IDE) y la modificación de impuestos especiales a otros productos como a bebidas saborizadas y alimentos con alto contenido calórico, por mencionar lo más relevante (Gobierno de México, 2013). Estos cambios, más las eficiencias en la Administración Tributaria permitieron un incremento de 1.1 puntos del PIB en el ISR para 2015.

Además, el Poder Ejecutivo firmó el Acuerdo de Certidumbre Tributaria, en el que se comprometió a no incrementar impuestos hasta el término de su mandato en 2018 (sHCP, 2014), compromiso que se refrendó en el cambio de gobierno. En 2016 se abolió el impuesto fijo comercial, pero se limitó la deducibilidad de salarios (Banco Mundial, 2019). Después de la reforma, el país no ha tenido un incremento similar en los ingresos tributarios, por el contrario, contrastándolo con el ańo anterior, se redujeron en 0.5 puntos en 2017 y permanecieron en el mismo nivel en 2018.

\section{Priorización y eficiencia del gasto público}

Para que el gasto público se realice con eficiencia y eficacia es necesaria una planeación integral y de largo plazo, donde se reconozcan las necesidades de gasto básicas e ineludibles para que se logren las metas como país. Orientar la política de gasto hacia los sectores productivos y al desarrollo con equidad, permitirá que en el mediano y largo plazo se incentive el crecimiento (Ros, 2015). Como consecuencia traerá mayores ingresos públicos y una amplia- 
ción espacio fiscal. Además, los gastos que han sido ineludibles para el gobierno deberán ser disminuidos y contenidos, para permitir una reorientación de los recursos.

Hasta antes del 2015, el gasto público en México se había definido de manera inercial (sHCP, 2015). La práctica usual era la de agregar y quitar programas con base en el presupuesto del ańo anterior. Ante la caída de los precios del petróleo, ocurrida a finales del 2014, el incremento en las tasas de interés por parte de Estados Unidos y las presiones del gasto público por el pago de pensiones y los gastos no programables, el gobierno mexicano se vio obligado a plantear una reestructura del gasto en enero del 2015. Dicha práctica le permitiría encontrar un mayor espacio fiscal, para cumplir con la operación del gobierno y aplicar políticas públicas emblemáticas de la gestión.

La reestructura del gasto público con perspectiva base 0 , planteada por el Ejecutivo federal pretendía una mejora en la administración pública a través de la eficiencia en procesos y reducción de estructuras administrativas. Se eliminaron programas que presentaban duplicidad dentro de la estructura central y se pretendía privilegiar los proyectos de inversión (sHCP, 2015). Sin embargo, esta revisión del gasto dejó de lado la evaluación del presupuesto con medidas de eficiencia y eficacia que permitieran ampliar el espacio fiscal de manera sostenida.

Las acciones de 2015 resultaron insuficientes para la aplicación de política de subsidios y transferencias, ya que para 2016 este gasto se redujo en 0.2 puntos del PIB, al igual que los servicios personales (véase gráfica 5). La liberación de recursos, derivada de la reestructura del gasto, se orientó a cubrir el gasto no programable y otros gastos de capital que no consideran la inversión física, rubros considerados ineludibles en la metodología propuesta en esta investigación. Para 2016, el gasto en inversión financiera y en deuda pública se incrementaron en 1 y 0.14 puntos PIB, respectivamente. Por fortuna, el remanente del Banco de México, recibido en 2016, permitió seguir con la tendencia inercial. Para 2017, a pesar de contar con otro remanente, el gasto público total se contrajo más de 2 puntos PIB, afectando principalmente al gasto de inversión.

Para ampliar el espacio fiscal es necesaria una reestructura del presupuesto público gradual y de mediano plazo. El Banco Mundial (2004) y Villa (2015) proponen que primero se reconozcan los compromisos ineludibles para guiarlos a su modificación al paso de algunos años. Segundo, los programas sujetos a reglas de operación y con una población beneficiaria definida deberán ser revisados bajo la perspectiva de eficiencia y eficacia para modificarlos en el mediano plazo. Tercero, los recursos no comprometidos bajo las dos perspec- 
Gráfica 5. Clasificación del gasto público según modalidad (porcentaje del PIB)

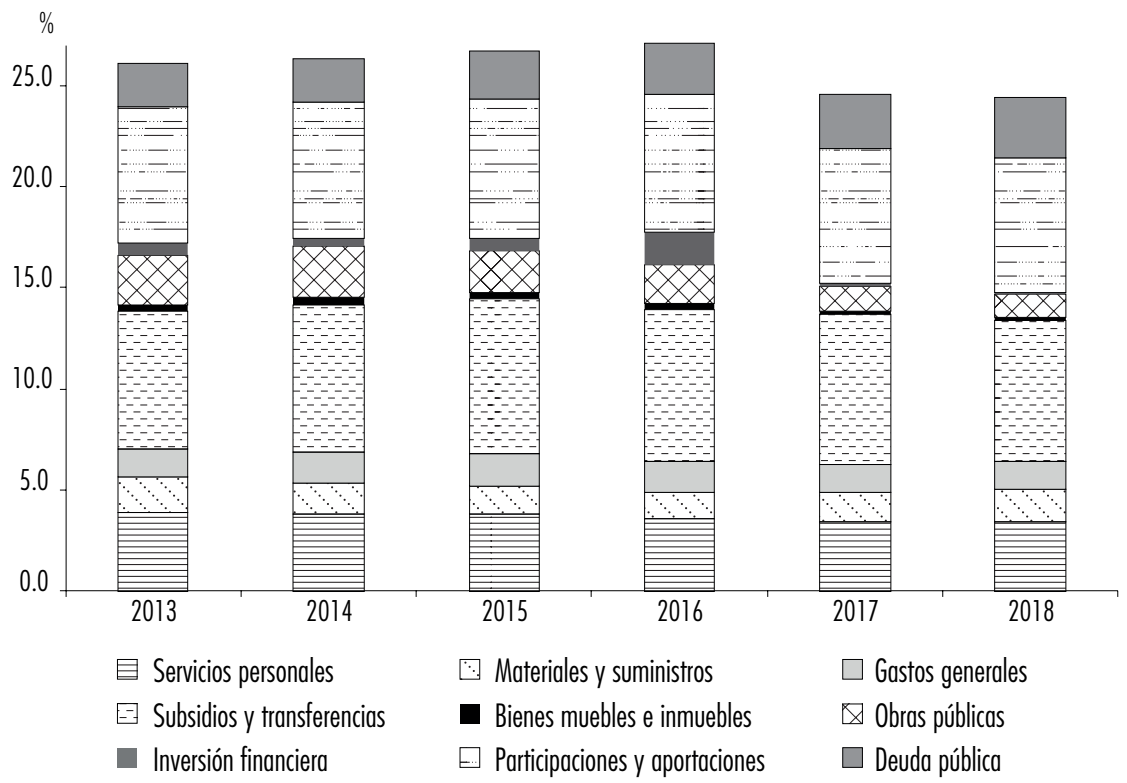

Nota: se muestra el gasto neto de transferencias.

Fuente: elaboración propia con datos de la SHCP (2013-2018). Cuenta de la Hacienda Pública Federal.

tivas anteriores y que no implican a la provisión de servicios básicos podrán ser reorientados para aplicar política de coyuntura. Aunque no es una solución rápida que libere la mayoría de los recursos permitirá orientar el gasto público de manera responsable y en favor del desarrollo. Además, resulta pertinente explorar la ampliación del espacio fiscal a través de los recursos administrados por las entidades federativas y los municipios, lo cual tendrá impacto a nivel federal. Aunque la estimación del espacio fiscal en los estados queda fuera del alcance de este trabajo, Pérez y Villarreal (2019) proponen una serie de indicadores para su evaluación.

\section{CONCLUSIONES}

La búsqueda del espacio fiscal para la aplicación de políticas públicas en favor del desarrollo de los países es un problema que enfrentan todos los gobiernos. El término por sí mismo es objeto de debate, pues puede ser abordado desde diversos ángulos. Por un lado, se encuentra la definición del espacio fiscal en 
función del endeudamiento. Por otro, se encuentran quienes aluden a la sostenibilidad de largo plazo; y quienes refieren a la reestructuración y priorización del gasto público para el desarrollo. Esta investigación propone una definición alterna de espacio fiscal, el cual se entiende como la diferencia entre ingresos totales y gastos ineludibles.

El presente trabajo muestra el lado operativo de la metodología que propone, y cuantifica los recursos que podría disponer el gobierno mexicano en un corto plazo. Sin embargo, el marco presentado pudiera tener otros usos. Por ejemplo, formar parte de un modelo estructural donde se considere al crecimiento como endógeno haciendo una conexión entre las decisiones microeconómicas y variables macroeconómicas que inciden en la política fiscal.

Con la aplicación de la metodología planteada se encuentra que México, a nivel federal, tiene un espacio fiscal menor a 1 punto del PIB en el corto plazo. De mantenerse los gastos ineludibles y los ingresos contantes y sin cambios estructurales, el espacio fiscal en el mediano plazo será de 5 puntos del piB. Este trabajo reconoce que existe la oportunidad de ampliar el espacio fiscal a través del pacto fiscal federal con las entidades federativas, dejando el tema a futuras investigaciones.

Conocer el espacio fiscal con el que cuentan los países permite impulsar acciones para salir de la trampa del lento crecimiento y alta desigualdad, como lo planteó Ros (2015). Con la ampliación del espacio fiscal, a través de distintas medidas en materia de ingresos, gasto, deuda y ayuda internacional, se podría subsanar el déficit de infraestructura, mejorar los servicios públicos, potenciar la inversión privada y con ello buscar el crecimiento de los países, especialmente, de México. Queda para futuros trabajos la orientación de recursos para el desarrollo que considere los problemas de economía política pertinentes, así como la aplicación metodológica a otros países y entidades federativas en México.

\section{BIBLIOGRAFÍA}

Aguzzoni, L. (2011), The concept of fiscal space and its applicability to the development of social protection policy in Zambia, International Labour Organization, ess Paper Series No. 28. Recuperado de <https://www.ilo. org/secsoc/information-resources/publications-and-tools/Workingpapers/ WCMS_207674/lang--en/index.htm>

Aizenman, J. y Jinjarak, Y. (2010), De facto fiscal space and fiscal stimulus: Definition and assessment, National Bureau of Economic Research, Working Paper No. 16539, DOI <http://dx.doi.org/10.3386/w16539> 
Alm, J. (2012), "Designing alternative strategies to reduce tax evasion", en Pickhardt y Prinz (eds.), Tax evasion and the shadow economy, Edward Elgar Publishing, DOI <https://doi.org/10.4337/9781781006740>

Awasthi, R. y Bayraktar, N. (2014), Can tax simplification help lower tax corruption?, Banco Mundial, DOI <https://doi.org/10.1596/1813-94506988>

Banco Mundial (2004), México-Análisis del gasto público: revisión del gasto público. Recuperado de <http://documentos.bancomundial.org/curated/ es/533891468120541722/Revision-del-gasto-publico>

(2016), México-Análisis del gasto público: revisión del gasto público. Recuperado de <http://documentos.bancomundial.org/curated/es/44488 $1472615646659 / \mathrm{M} \% \mathrm{C} 3 \%$ A9xico-Revisi\%C3\%B3n-del-gastop\%C3\%BAblico>

(2019), "Paying taxes", Doing Business. Recuperado de <https://www. doingbusiness.org/en/data/exploretopics/paying-taxes/reforms >

Celasun, O., Grigoli, F., Honjo, K., Kapsoli, J., Klemm, A., Lissovolik, B., Luksic, J., Moreno-Badia, M., Pereira, J., Poplawski-Ribeiro, M., Baoping, S. y Ustyugova, Y. (2015), Fiscal policy in Latin America: Lessons and legacies of the global financial crisis, IMF Staff Discussion Notes 15/6, FMI. Recuperado de <https://www.imf.org/external/pubs/ft/sdn/2015/sdn1506.pdf>

Cetrángolo, O., Jiménez, J. y Ruiz del Castillo, R. (2010), Rigidities and fiscal space in Latin America: A comparative case study, ECLAC. Recuperado de <https://repositorio.cepal.org/handle/11362/5470>

Development Committee (2006, April), Fiscal policy for growth and development: An interim report, Development Committee Meeting, Washington DC. Recuperado de <http://siteresources.worldbank.org/DEVCOMMIN T/Documentation/20890698/DC2006-0003(E)-FiscalPolicy.pdf>

Estevão, M. (2019), Cuatro maneras en que las economías de ingreso bajo pueden aumentar los ingresos fiscales sin afectar el crecimiento, Banco Mundial. Recuperado de <https://blogs.worldbank.org/es/voices/cuatro-mane ras-en-que-las-economias-de-ingreso-bajo-pueden-aumentar-los-in gresos-fiscales?cid=ECR_E_NewsletterWeekly_ES_EXT\&deliveryNa me=DM49251>

FMI (2017), IMF fiscal monitor: Achieving more with less, International Monetary Fund. Recuperado de <https://www.imf.org/en/Publications/FM/ Issues/2017/04/06/fiscal-monitor-april-2017>

(2018), Assessing fiscal space: An update and stocktaking, International Monetary Fund. Recuperado de <https://www.imf.org/en/Publications/ Policy-Papers/Issues/2018/06/15/pp041118assessing-fiscal-space> 
Ghosh, A., Ostry, J. y Qureshi, M. (2013), "Fiscal space and sovereign risk pricing in a currency union", Journal of International Money and Finance, vol. 34, DOI <https://doi.org/10.1016/j.jimonfin.2012.11.008>

Gobierno de México (2013), Reforma de la Hacienda Pública. Recuperado de <https:/www.gob.mx/cms/uploads/attachment/file/66458/7_Hacenda ria.pdf $>$

Heller, M. (2005), Understanding fiscal space, Policy Discussion Paper No. 05/4 FMI. Recuperado de <https://www.imf.org/en/Publications/IMFPolicy-Discussion-Papers/Issues/2016/12/31/Understanding-Fiscal-Space-18065>

International Labour Office (2012), Creating fiscal space for PEPs. Towards the right to work: a guidebook for designing innovative public employment programmes. Recuperado de <https://www.ilo.org/global/topics/employmentintensive-investment/publications/WCMS_559267/lang--en/index.htm>

Kose, M. A., Kurlat, S., Ohnsorge, F. y Sugawara, N. (2017), A cross-country database of fiscal space, The World Bank. Recuperado de <http://documents.worldbank.org/curated/en/601211501678994591/pdf/WPS8157. pdf>

Llop, M. y Flores, M. (2005), "Modelos multisectoriales de equilibrio general aplicado en España: una revisión”, Estudios de Economía Aplicada, vol. 23, núm. 2. Recuperado de <https://dialnet.unirioja.es/servlet/ articulo? codigo $=1250451>$

Lofgren, H. (2013), Creating and using fiscal space for accelerated development in Liberia, Banco Mundial. Recuperado de <http://documents.worldbank. org/curated/en/660331468265793638/Creating-and-using-fiscal-spacefor-accelerated-development-in-Liberia>

Núñez-Rodríguez, G. (2015), "Modelo de equilibrio general aplicado para México y análisis de impuestos a la extracción de hidrocarburos", Ensayos Revista de Economía, vol. 34, núm. 1. Recuperado de <https://ideas.repec. org/a/ere/journl/vxxxivy2015i1p35-74.html>

oCDE (2018), Base de datos global de estadísticas tributarias. Recuperado de $<$ https://www.oecd.org/tax/tax-policy/base-de-datos-global-de-estadisticas-tributarias.htm>

Ostry, J. D., Ghosh, A., Kim, J. y Qureshi, M. (2010), Fiscal Space. IMF Staff Position Note 10/11, FMI. Recuperado de <https://www.imf.org/ en/Publications/IMF-Staff-Position-Notes/Issues/2016/12/31/Fiscal-Space-23726> 
Pedauga, L., Sáenz, F. y Velázquez, A. (2012), “Simulación de un modelo de equilibrio general computable para Venezuela", El Trimestre Económico, vol. 79, núm. 314, DOI <http://dx.doi.org/10.20430/ete.v79i314.148>

Pérez, N. y Villarreal, H. (2019), "El espacio fiscal en los estados", en A. M. Velázquez, Los incentivos perversos del federalismo fiscal mexicano, México, Fondo de Cultura Económica.

Perotti, R. (2007), Fiscal policy in developing countries: A framework and some questions, Banco Mundial. Recuperado de <http://documents.worldbank. org/curated/en/108941468138570564/Fiscal-policy-in-developing-countries-a-framework-and-some-questions $>$

Pommier, S. (2015), Public debt sustainability in advanced economies: A stochastic simulation of fiscal spaces after the 2008 financial crisis. Recuperado de <https://afse2015.sciencesconf.org/61503/document>

Rafiq, A. (2019), Is your financial services organization ready for changes to the international tax framework? Recuperado de https://www.pwc.com/gx/en/ services/tax/publications/fs-organisation-ready-for-change-internationaltax-framework.html?WT.mc_id=CT1-PL52-DM1-TR2-LS2-ND30TTAT3-CN_FS.Tax.Blog-Paid.TW

Ros, J. (2008), "La desaceleración del crecimiento económico en México desde 1982", El Trimestre Económico, vol. 75 (3), núm. 299, DoI <http:// dx.doi.org/10.20430/ete.v75i299.411>

(2013), Algunas tesis equivocadas sobre el estancamiento económico de México, México, El Colegio de México A.C.

(1 de julio de 2014), Réplica, Nexos. Recuperado de <https://www. nexos.com. $\mathrm{mx} / \mathrm{p}=21690>$

(2015), ¿Cómo salir de la trampa del lento crecimiento y alta desigualdad?, México, El Colegio de Mexico A.C.

(2019), "Los retos de la política económica", Economía UNAM, vol. 16, núm. 46, DoI <https://doi.org/10.22201/fe.24488143e.2019.46.419>

Roy, R., Heuty, A. y Letouzé, E. (2007), Fiscal space for what? Analytical issues from a human development perspective, Programa de las Naciones Unidas para el Desarrollo. Recuperado de <https:/www.undp.org/content/dam/ aplaws/publication/en/publications/poverty-reduction/poverty-website/ fiscal-space-for-what/FiscalSpaceforWhat.pdf>

Secretaría de Gobernación (30 de abril de 2014), Programa de Cooperación Internacional para el Desarrollo 2014-2018, Diario Oficial de la Federación. Recuperado de <http://www.dof.gob.mx/nota_detalle_popup.php?c odigo $=5342827>$ 
Secretaría de Hacienda y Crédito Público (shcp) (2013-2018), Cuenta de la Hacienda Pública Federal. Recuperado de <https://www.finanzaspublicas. hacienda.gob.mx/es/Finanzas_Publicas/Cuenta_Publica>

(2014), Acuerdo de Certidumbre Tributaria. Recuperado de <http:// www.shcp.gob.mx/Biblioteca_noticias_home/acuerdo_gabinete_mexico_prospero_27022014.pdf>

(2015), La SHCP anuncia medidas de responsabilidad fiscal para mantener la estabilidad. Comunicado de Prensa 07/2015. Recuperado de $<\mathrm{http}: / /$ www.shcp.gob.mx/SALAPRENSA/doc_comunicados_prensa/2015/enero/comunicado_007_2015.pdf>

(2019), Estadisticas oportunas. Recuperado de <http://presto.hacienda.gob.mx/EstoporLayout/estadisticas.jsp>

Secretaría de Relaciones Exteriores (2018), Programa de Cooperación Internacional para el Desarrollo 2014-2018. Informe de Avance y Resultados 2018. Recuperadode<https://transparencia.sre.gob.mx/amexcid/images/pdf/pro cid/logros-2018-del-PROCID.pdf>

Sobarzo, H. (2009), Reforma Fiscal en México: un modelo de equilibrio general, Centro de Estudios de las Finanzas Públicas. Recuperado de <https:// www.cefp.gob.mx/intr/edocumentos/pdf/cefp/2009/cefp0152009.pdf >

Vázquez, C. (2019), Deuda pública. Proyección al cierre de 2019, Centro de Investigación Económica y Presupuestaria A.C. Recuperado de <https:// ciep.mx/deuda-publica-proyeccion-al-cierre-de-2019/>

Villa, S. (2015), Restructuración del gasto público con perspectiva "base cero", Centro de Investigación Económica y Presupuestaria, A.C. Recuperado de $<$ https://ciep.mx/reestructuracion-del-gasto-publico-con-perspectiva-ba se-cero/> 